Brain Topography manuscript No.

(will be inserted by the editor)

\title{
The joint use of the tangential electric field and surface Laplacian in EEG classification
}

\author{
C. G. Carvalhaes - J. Acacio de Barros . \\ M. Perreau-Guimarães · P. Suppes
}

the date of receipt and acceptance should be inserted later

\begin{abstract}
We investigate the joint use of the tangential electric field and the surface Laplacian derivation as a method to improve the classification of EEG signals. We considered five classification tasks to test the validity of such approach. In all five tasks, the joint use of the components of the tangential electric field and the surface Laplacian outperformed the scalar potential. The smallest effect occurred in the classification of a mental task, wherein the average classification rate was improved by 0.5 standard deviations. The largest effect was obtained in the classification of visual stimuli and corresponded to an improvement of 2.1 standard deviations.
\end{abstract}

Keywords Scalp electric field · EEG classification · Surface Laplacian · EEG brain mapping.

\section{Introduction}

Advanced techniques of analysis and interpretation of the EEG signals have grown substantially over the years, with special attention directed to the problems of low spatial resolution and the choice of physical reference. Performing the surface Laplacian differentiation of scalp potentials has proved

C. G. Carvalhaes $(\varangle) \cdot$ M. Perreau-Guimarães · P. Suppes

Suppes Brain Lab, 220 Panama St, Ventura Hall, Stanford, CA 94305-4115

E-mail: claudioc@stanford.edu,mpguimaraes@stanford.edu,psuppes@stanford.edu

J. Acacio de Barros

Liberal Studies, San Francisco State University, 1600 Holloway Ave, San Francisco, CA 94312

E-mail: barros@sfsu.edu 
to be an efficient method to address both issues (Hiorth, 1975; Perrin et al, 1989; He and Cohen, 1992; He et al, 1993; Babiloni et al, 1995; Yao, 2002;

Nunez and Srinivasan, 2006). The surface Laplacian operation is reference-free and many studies have suggested that it provides a more accurate representation of dura-surface potentials than conventional topography (Nunez and Pilgreen, 1991; Nunez et al, 1991; Nunez and Westdorp, 1994; Nunez et al, 1994; Srinivasan et al, 1996; Nunez and Srinivasan, 2006). Motivated by this observation, numerous experimental studies of both clinical and theoretical interest have been successfully conducted, such as those of Babiloni et al (1999, 2000, 2001, 2002); Chen et al (2005); Besio et al (2006); Kavser and Tenke (2006a), b); Koka and Besio (2007); Bai et al (2008); Besio et al (2009).

In physical terms, the surface Laplacian of the scalp potential is a measure of local effects of geometry and boundary conditions on the normal component of the underlying current density. This follows directly from the quasistatic continuity of the current density, which implies that any change in flux normal to a surface causes a lateral divergence of flow lines, which may be expressed mathematically as the surface Laplacian of the surface potential. This relationship between the surface Laplacian of the potential and the normal component of the current density results in a spatial filtering property that is responsible for the majority of practical applications of the Laplacian technique. But unless reliable information is available about the physical process underlying the EEG, relying exclusively on the behavior of the normal component of the current density may imply the neglect of potentially valuable information encoded in other spatial components. This observation was a compelling reason to undertake the present work. Thus, in our approach we jointly consider the surface Laplacian of the scalp potential and the tangential components of the scalp electric field to classify EEG signals.

The rationale for this combination is that the electric field is also locally related to the current density by Ohm's law. Each spatial component of the electric field expresses the (negative) rate of change of the scalar potential in that direction, but because the EEG is only recorded along the scalp, we cannot estimate the field component normal to the scalp surface directly from the data. The use of the surface Laplacian to represent this spatial component is not new in the literature. For instance, He et al (1995) discussed the physical existence of the normal component of the electric field just out of the body surface and used its analytic relationship with the surface Laplacian of the potential to construct a surface-charge model to represent bioelectrical sources 
inside the body. In the Appendix, we use similar considerations to explain the connection between these quantities at electrode sites on a spherical scalp model.

All computations in our work were performed by means of regularized splines on the sphere. We evaluated the practical effect of the joint approach on five classification tasks, derived from experiments on language, visual stimuli, and a mental task. The results in terms of effect sizes showed an optimistic prospect for further developments and applications.

\section{Methods}

Experimental Procedures

All data used in our study were previously obtained in our laboratory as part of experiments on language, vision, and imagination. We label such experiments as Exp. I, Exp. II, and Exp. III, and the subjects who took part in them as S1, S2, S3, $\cdots$, but S1 of Exp. I was not necessarily the same as S1 of Exp. II, and so on. Exp. I encompassed three distinct classification tasks and Exp. II and III one classification task each.

\section{Exp. I: 32 consonant-vowel syllables}

This experiment emerged from Rui Wang's doctoral work (Wang, 2011) and is described in detail in Wang et al (2012), to which the reader is referred for further details. The focus was on the identification of brain patterns related to listening to a set of English phonemes having traditional phonological features of consonants (voicing, continuant, and place of articulation) and vowels (height and backness). The stimuli consisted of the sounds of 32 phonemes ( 8 consonants $\times 4$ vowels) formed from pairwise combinations of the consonants $/ \mathrm{p} /, / \mathrm{t} /, / \mathrm{b} /, / \mathrm{g} /, / \mathrm{f} /, / \mathrm{s} /, / \mathrm{v} /, / \mathrm{z} /$, and the vowels $/ \mathrm{i} /$ (as in meet), /ïœ $œ /$ (cat), $/ \mathrm{u} /$ (soon), and $/ \mathrm{a} /$ (spa). These vowels were selected also for being maximally separated in the American-English vowel space, which presumably facilitates classification. All phonemes were uttered by a male native speaker of English and recorded in audio files at $44.1 \mathrm{kHz}$ sampling rate. Each syllable was repeated 7 times to produce a variation of pronunciation as commonly occurs in human languages. This resulted in a total of $7 \times 32=224$ audio files for presentation. 
Four adult subjects (S1-S4), 1 male, participated in this experiment, all reporting no history of hearing problems. The auditory stimuli were presented to participants in random order via a stereo computer speaker. Each participant was instructed to listen carefully to the sounds and try to comprehend them, but no response was required. The stimulus presentations were grouped into multiple sessions of 896 trials (4 repetitions of 224 sounds at random). The trial length, measured from the onset of one stimulus to the onset of the next, had 1,000 ms duration, so that each session lasted approximately $15 \mathrm{~min}$. There was a short break after each block of 56 trials, and the participant could control the length of the break by pressing the spacebar. The number of trials collected from the participants were: 7,168 (S1), 3,584 (S2), 6,272 (S3), and 4,480 (S4).

EEG signals were recorded at $1,000 \mathrm{~Hz}$ sampling rate, using a 128-channel Geodesic Sensor Net (Figure1) on EGI's Geodesic EEG system 300. There were 124 monopolar channels with a common reference $\mathrm{Cz}$ and 2 bipolar reference channels for eye movements.

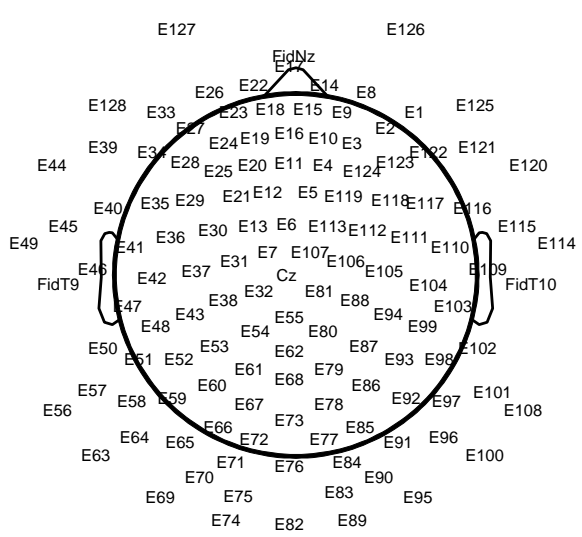

Fig. 1 Electrode montage used in Exp. I.

Exp. II: 9 shape-color images

In this experiment researchers from our Lab investigated the brainwave representation of nine two-dimensional images, formed by pairwise combinations of three geometric shapes (circle, square, and triangle) and three colors (red, green, and blue). These images were presented to participants on a 17-inch LED computer screen using the commercial software Presentation. All shapes 
had approximately the same area of $100 \mathrm{~cm}^{2}$, and the physical luminosity was adjusted for each object and color to appear the same at $60 \mathrm{~cm}$ from the screen, but no attempt was made to adjust images to each participant, such that the subjective perception of luminosity was the same for all colors and each participant. The distance from the participant' eyes to the screen was approximately $60 \mathrm{~cm}$ and the visual angle was $2.3-3.3^{\circ}$. Each presentation lasted $300 \mathrm{~ms}$ and was followed immediately by an interval of $700 \mathrm{~ms}$, during which a fixation cross $\left({ }^{\prime}+\right.$ ') was shown at the center of a blank screen. A stimulus appeared randomly and with equal probability every $1,000 \mathrm{~ms}$.

Seven adults (S1-S7), 3 female, agreed to participate in this experiment, all having normal or corrected to normal vision. Participants were instructed to remain relaxed and motionless, and to keep eyes fixed at the center of the screen during presentations. They were seated comfortably on a chair in a dimly lit sound-attenuated booth and responded to 2,700 trials timelocked to stimulus presentations. The presentations were divided in blocks of 20 trials and the participant could control the duration of the breaks via the spacebar. Halfway through the experiment a modified break message was displayed informing the participant that the experiment had passed its halfway point.

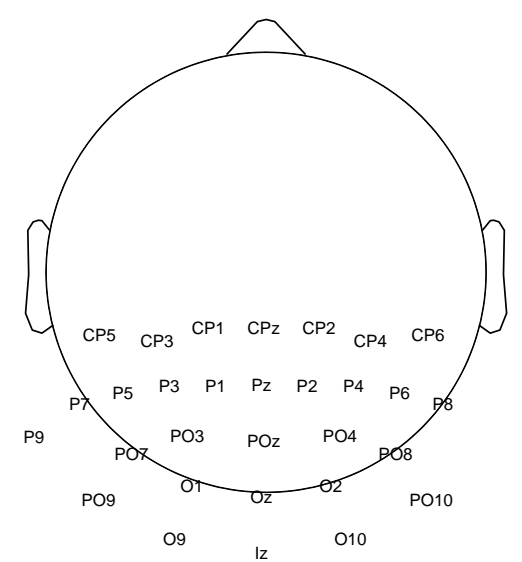

Fig. 2 Electrode montage used in Experiment II.

Exp. III: 2-class imagery task

The third experiment was previously described in Carvalhaes et al (2009) and Carvalhaes and Suppes (2011). Eleven participants (S1-S11) were randomly 
presented on every other trial either a visual "stop" sign, flashed on a 17-inch LED computer screen, or the sound of the English word "go", via computer speaker. The "go" sound was uttered by a male native speaker of English and recorded in an audio-isolated cabin using a professional microphone interfaced with a computer via a Sound Blaster II (Creative Labs) sound card at $44.1 \mathrm{kHz}$ sampling rate (24 bits). Stimuli were delivered using the Presentation software. The "go" sound was delivery via high fidelity PC speakers at the level of normal conversation. Each stimulus presentation lasted $300 \mathrm{~ms}$, and was followed by a period of $700 \mathrm{~ms}$ of blank screen. Immediately after this period a fixation cross ('+') was shown at the center of the screen for $300 \mathrm{~ms}$.

Eleven subjects participated in this experiment, all adults reporting normal vision and normal hearing. They were comfortably seated in a chair at a distance of approximately $60 \mathrm{~cm}$ from a computer screen and $100 \mathrm{~cm}$ from the speaker. For one group (S1-S7) the participants were instructed to form a vivid mental image of the stimulus previously presented, for another group (S8-S11) they were asked to form a mental image of the alternative stimulus, i.e., if the last stimulus was the "stop" sign, then they should imagine the "go" sound, and vice versa. Participants' imagining was followed by another $700 \mathrm{~ms}$ of blank screen, after which the trial ended. A single session of 600 trials was recorded for each participant. The session was divided into thirty 20 -trial blocks, with regular breaks controlled by participants via the spacebar. Each trial lasted 2,000 ms, but only the last 1,000 ms of each trial corresponding to the imagination task was used for our analysis.

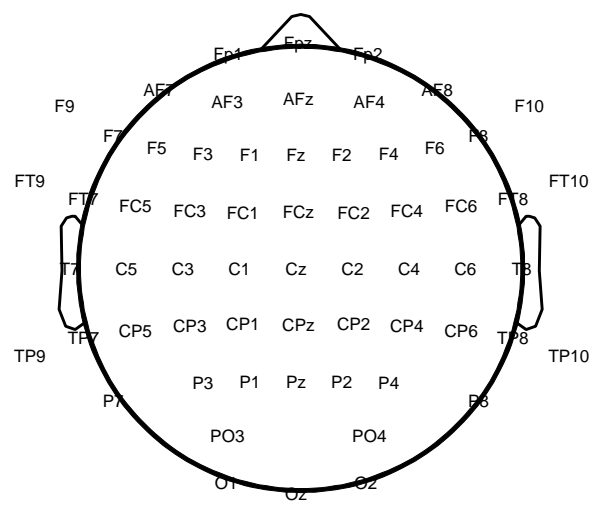

Fig. 3 Electrode montage used in Experiment III. 
Data collection and preprocessing

Data collection started after participants were given the opportunity to practice the required tasks. The recording apparatus changed from one experiment to another. Exp. I was carried out using EGI's Geodesic EEG system with 128 monopolar channels referenced to the vertex electrode $(\mathrm{Cz})$ and with a ground electrode placed on the forehead (also for Exp II and III). The electrode locations for this experiment are illustrated in Figure1, In Exp. II signals were recorded using a 32-channel NeuroScan system with linked earlobe reference $(\mathrm{Ag}-\mathrm{AgCl}$ electrodes). Due to the low number of channels available on this device - and in view of the need for a reasonable density of electrodes to accurately estimate the electric field in the region of interest V1 (Mikkulainen, 2005) - the measurement electrodes were all placed in the back part of the head, as depicted in Figure2. We remark that there was no particular reason for choosing P9 instead of P10 in this montage. The electrode distribution was asymmetric and P10 was not included as well just because of the small number of channels that were available to perform this experiment. Exp. III used a 64-channel Neuroscan system, following the 5\% system of Oostenveld and Praamstra (2001), but not including electrodes Nz, AF1, AF2, AF5, AF6, T9, T10, P9, P5, P6, P9, P10, PO, or I; the reference being as in Exp. II. Figure 3 shows the electrode distribution for this experiment.

The signals were passed through a band-pass filter in the range $0.1-300 \mathrm{~Hz}$ plus a $60 \mathrm{~Hz}$ notch filter, and digital conversion was performed at a $1 \mathrm{kHz}$ sampling rate. To reduce features, we carried out offline decimation at 16:1 ratio, thus setting the Nyquist frequency at $31.25 \mathrm{~Hz}$. Additionally, we removed unwanted low-frequency components by applying a high-pass filter of $1 \mathrm{~Hz}$. Finally, we mathematically referenced the decimated signals to the average reference voltage to reduce biases in the analysis of the potential distribution (Bertrand et al, 1985; Murrav et al, 2008). This step had no effect on the tangential field and the surface Laplacian derivation, for they are reference-free quantities (He et al , 1993, 1995; Nunez and Srinivasan, 2006).

The signals were visually inspected, but no trial was removed. Thus, robustness to outliers and artifacts was also tested in the classification. In order to enhance the signal-to-noise ratio, we averaged same-class trials over small groups of trials before classification. We fixed the number of samples per average trial according to the amount of classes and the total number of trials available in the experiment. With this constraint in mind, the number of sam- 
ples per average trial was set to: 12 (Exp. I, 8 initial consonants); 5 (Exp. I, 32 syllables); 20 (Exp. I, 4 vowels); 5 (Exp. II); and 5 (Exp. III).

Numerical procedure

For convenience, we adopted spherical coordinates $(r, \theta, \varphi)$, where $r$ stands for radial distance and $\theta$ and $\varphi$ are the angular coordinates, with $\theta$ increasing down from the vertex and $\varphi$ increasing counterclockwise from the nasion. The scalar potential, the tangential components of the electric field, and the surface Laplacian of the potential were denoted by $\Phi_{s}^{\text {scalp }}, E_{\theta}^{\text {scalp }}, E_{\varphi}^{\text {scalp }}$, and $\nabla_{s}^{2} \Phi_{s}^{\text {scalp }}$. The mathematical expressions for these quantities are shown in the Supplementary Material in terms of partial derivatives of $\Phi_{s}^{\text {scalp. }}$. To obtain these quantities we fitted $\Phi_{s}^{\text {scalp }}$ with a spline interpolant and then applied the partial derivatives analytically to the interpolant. This computation was carried out using $\lambda$-correction to attenuate the effect of spatial noises on the estimates (Wahba, 1990; Babiloni et al, 1995).

Using splines we can calculate partial derivatives at a very low computational cost. Assume an instantaneous distribution of scalp potentials $\left\{V_{1}, \cdots, V_{N}\right\}$, sampled at electrode locations $\mathbf{r}_{1}, \cdots, \mathbf{r}_{N}$ at a time $t$. The spline interpolant that fits or smooths this distribution is defined by

$$
f_{\lambda}(\mathbf{r})=\sum_{j=1}^{N} c_{j}\left\|\mathbf{r}-\mathbf{r}_{j}\right\|^{2 m-3}+\sum_{\ell=1}^{M} d_{\ell} \phi_{\ell}(\mathbf{r}),
$$

where $m$ is an integer greater than $2, M=\left(\begin{array}{c}m+2 \\ 3\end{array}\right)$ is subject to $M<N$, $\phi_{1}, \cdots, \phi_{M}$ are linearly-independent polynomials in $\mathbb{R}^{3}$ of degree less than $m$, and $c_{j}$ and $d_{j}$ are data-dependent parameters. In order to avoid the magnification of high-frequency spatial noises, we introduce a regularization parameter, $\lambda$, such that (Wahba, 1990; Babiloni et al, 1995)

$$
\left(\begin{array}{cc}
\mathbf{K}+N \lambda \mathbf{I} & \mathbf{T} \\
\mathbf{T}^{\prime} & 0
\end{array}\right)\left(\begin{array}{l}
\mathbf{c} \\
\mathbf{d}
\end{array}\right)=\left(\begin{array}{l}
\mathbf{v} \\
\mathbf{0}
\end{array}\right)
$$

where $(\mathbf{K})_{i j}=\left\|\mathbf{r}_{i}-\mathbf{r}_{j}\right\|^{2 m-3},(\mathbf{T})_{i j}=\phi_{j}\left(\mathbf{r}_{i}\right), \mathbf{c}=\left(c_{1}, \cdots, c_{N}\right)^{\prime}, \mathbf{d}=\left(d_{1}, \cdots, d_{M}\right)^{\prime}$, and $\mathbf{v}=\left(V_{1}, \cdots, V_{N}\right)^{\prime}$. As explained in Carvalhaes and Suppes (2011) and Carvalhaes (2013), the system (2) is singular on a spherical surface, so that we can not obtain $\mathbf{c}$ and $\mathbf{d}$ by just inverting this system. Instead, following 
Carvalhaes and Suppes (2011) we factorize $\mathbf{T}$ as

$$
\mathbf{T}=\left(\mathbf{Q}_{\mathbf{1}}, \mathbf{Q}_{\mathbf{2}}\right)\left(\begin{array}{c}
\mathbf{R} \\
O
\end{array}\right),
$$

where $\mathbf{Q}_{1} \in \mathbb{R}^{N \times M}$ and $\mathbf{Q}_{2} \in \mathbb{R}^{N \times(N-M)}$ are orthonormal and $\mathbf{R} \in \mathbb{R}^{M \times M}$ is upper triangular, and introduce the auxiliary matrices

$$
\begin{aligned}
& \mathbf{C}_{\lambda}=\mathbf{Q}_{2}\left[\mathbf{Q}_{2}^{\prime}(\mathbf{K}+N \lambda \mathbf{I}) \mathbf{Q}_{2}\right]^{-1} \mathbf{Q}_{2}^{\prime}, \\
& \mathbf{D}_{\lambda}=\mathbf{R}^{+} \mathbf{Q}_{1}^{\prime}\left(\mathbf{1}-\mathbf{K} \mathbf{C}_{\lambda}-N \lambda \mathbf{C}_{\lambda}\right),
\end{aligned}
$$

where $\mathbf{R}^{+}$is the pseudo-inverse of $\mathbf{R}$.

Let $\mathbf{e}_{\theta, \lambda}, \mathbf{e}_{\varphi, \lambda}$, and $\mathbf{l}_{\lambda}$ be $N$-dimensional vectors giving $E_{\theta}^{\text {scalp }}, E_{\varphi}^{\text {scalp }}$, and $\nabla_{s}^{2} \Phi_{s}^{\text {scalp }}$ at the electrode coordinates. These vectors can be obtained by linearly transforming the potential $\mathbf{v}$ as

$$
\begin{aligned}
\mathbf{e}_{\theta, \lambda} & =\left(\mathbf{K}_{\theta} \mathbf{C}_{\lambda}+\mathbf{T}_{\theta} \mathbf{D}_{\lambda}\right) \mathbf{v}=\mathbf{E}_{\theta, \lambda} \mathbf{v}, \\
\mathbf{e}_{\varphi, \lambda} & =\left(\mathbf{K}_{\varphi} \mathbf{C}_{\lambda}+\mathbf{T}_{\varphi} \mathbf{D}_{\lambda}\right) \mathbf{v}=\mathbf{E}_{\varphi, \lambda} \mathbf{v}, \\
\mathbf{l}_{\lambda} & =\left(\tilde{\mathbf{K}} \mathbf{C}_{\lambda}+\tilde{\mathbf{T}} \mathbf{D}_{\lambda}\right) \mathbf{v}=\mathbf{L}_{\lambda} \mathbf{v} .
\end{aligned}
$$

The analytic expressions for the matrices $\mathbf{K}_{\theta}, \mathbf{T}_{\theta}, \mathbf{K}_{\varphi}, \mathbf{T}_{\varphi}, \tilde{\mathbf{K}}$, and $\tilde{\mathbf{T}}$ are given in the supplementary material, along with a Matlab code implementation. The fact that $\mathbf{e}_{\theta, \lambda}, \mathbf{e}_{\varphi, \lambda}$, and $\mathbf{l}_{\lambda}$ are reference free implies that

$$
\mathbf{E}_{\theta, \lambda} \mathbf{v}_{\text {ref }}=\mathbf{E}_{\varphi, \lambda} \mathbf{v}_{\text {ref }}=\mathbf{L}_{\lambda} \mathbf{v}_{\text {ref }}=0,
$$

where $\mathbf{v}_{\text {ref }}=$ const. $\times(1, \cdots, 1)^{T}$ is a reference vector. That is, the columns of $\mathbf{E}_{\lambda}^{\theta}, \mathbf{E}_{\lambda}^{\varphi}$, and $\mathbf{L}_{\lambda}$ sum to zero, regardless of the value of $\lambda$. A skeptical reader is encouraged to use the Matlab code in the Supplementary Material to test this property.

\section{Classification procedures}

For statistical comparison, each experiment was classified using the potential, the surface Laplacian of the potential, the tangential electric field, and a combination of the last two into a three-dimensional vector. We carried out the classifications on single channels, using a 10-fold cross-validation on linear discriminant analysis (LDA) (Parra et al, 2008; Suppes et al, 2009). For this 
purpose, the data from each channel and waveform were rearranged in a rectangular matrix, with adjacent rows corresponding to adjacent trials and adjacent columns corresponding to adjacent time samples. Matrices representing vector quantities were given by the concatenation of the individual components.

Preliminary classifications were performed with a small number of trials, attempting to find a plausible range of values for the $\lambda$ parameter. This parameter regulates the trade-off between minimizing the squared error of the data fitting and smoothness (Wahba, 1990), thus influencing spatial differentiations and the classification rates. The overall most satisfactory results were obtained in a grid with 50 points, covering the interval $\lambda \in[0.001,100]$ in logarithm scale. Hence, for all tasks the classification of each channel was repeated 50 times, varying $\lambda$ across this interval.

To further improve the classification rates we applied principal component analysis (PCA) (Jolliffe, 2005), but only the $\lambda$-value yielding the highest classification rate was considered in this step. The classification of PCA-transformed data began with the classification of the first principal component, which ordinarily explains most of the variance of the data. The other components account for residual variance and were added in order of decreasing variance. For the purpose of pairwise comparison the same random sequence of trials was used in the classification of all waveforms.

\section{Statistical analysis}

We used effect sizes and confidence intervals to assess improvements in classification rates in comparison to the scalar potential. Effect size is a standard measure that addresses the practical relevance of differences in paired comparisons. Typically, it is calculated by dividing the difference between the means of two groups by the combined (pooled) standard deviation, i.e.,

$$
d=\frac{\mu_{A}-\mu_{B}}{s_{\text {pool }}}
$$

where $d$ stands for effect size (also known as Cohen's $d$ ), $\mu_{A}$ and $\mu_{B}$ are the mean values of the two groups, and $s_{\text {pool }}=\sqrt{\left(s_{A}^{2}+s_{B}^{2}\right) / 2}$ is the pooled standard deviation $\left(s_{A}^{2}\right.$ and $s_{B}^{2}$ are the respective variances). We used equation (17) with $B$ standing for the potential and $A$ standing for the tested waveform.

Intuitively, equation (7) expresses how many standard deviations separate the performance of two methods; the larger the effect size, the greater the 
performance of the tested method. A zero effect size indicates a failure in rejecting the null hypothesis of no difference between the methods. In other words, the effect size has the following practical application: it tells us not only whether the null hypothesis is being rejected, but also gives us a sense of the strength of this rejection. In contrast to null-hypothesis significance testing (often represented by a $p$-value), the effect size is not particularly sensitive to the sample size, and hence it can be compared across different studies, even though the number of samples is not the same.

In order to make our statistical comparison more reliable, we estimated a confidence limit around each effect size. Namely, the null hypothesis of no practical effect of the tested waveform was rejected at the $95 \%$ level of significance only if the estimated confidence interval did not include zero. The confidence interval of $d$ was estimated by the equation

$$
95 \% \mathrm{CI}=[d-1.96 \times \mathrm{SE}, d+1.96 \times \mathrm{SE}],
$$

where $\mathrm{SE}$ is the standard error between the paired rates from $A$ and $B$, given by

$$
\mathrm{SE}=\sqrt{\frac{2\left(1-r_{A B}\right)}{n_{p}}+\frac{d^{2}}{2\left(n_{p}-1\right)}}
$$

where $n_{p}$ is the number of participants in the experiment and $r_{A B}$ is the correlation coefficient for the paired rates (Becker, B. J., 1988; Nakagawa and Cuthill, 2007). Note that equation (9) depends on the sample size $n_{p}$. Large samples yield small errors and, consequently, narrow confidence intervals. In contrast, small samples provide less focused estimates of the effect size, but this cannot be mistaken as evidence for a null effect, as usually occurs when reporting $p$-values. This remark is particularly important to our study because $n_{p}$ was generally small, thus resulting in large confidence intervals.

\section{Results}

Classification rates

Tables 15 summarize the classification outcomes, showing the highest crossvalidation rate of each task, along with the best sensor. The surface Laplacian of the scalp potential and the tangential electric field are referred to by SL and EF, and their combination by SL \& EF. Bearing in mind the chance level of 
each task, generally the rates were remarkably good. In Table1 we show the classification rates for Exp. I using the initial consonants to define the eight classes. The combination of the surface Laplacian and the tangent electric field not only yielded the highest classification rate for all participants, but interestingly its best performance was achieved by locations on the primary auditory cortex A1 (Pickles, 2012) for all subjects. Averaged over all participants, this resulted in a improvement of $10.6 \%$ in comparison with the potential and $4.8 \%$ in relation to the surface Laplacian of the potential. The tangential electric field had a similar performance, but with individual classification rates being slightly smaller for all participants.

Table 1 Highest performance for the classification of the 8 initial consonants of Exp. I.

\begin{tabular}{|c|c|c|c|c|c|c|c|c|}
\hline \multirow[b]{2}{*}{ subject } & \multicolumn{2}{|c|}{ potential } & \multicolumn{2}{|c|}{$\overline{S L}$} & \multicolumn{2}{|c|}{$\overline{E F}$} & \multicolumn{2}{|c|}{ SL \& EF } \\
\hline & $\%$ & sensor & $\%$ & sensor & $\%$ & sensor & $\%$ & sensor \\
\hline S1 & 45.2 & E36 & 57.5 & E41 & 60.7 & E40 & 63.0 & E40,E41 \\
\hline S2 & 37.2 & E30 & 40.5 & E12 & 43.4 & E109 & 44.4 & E40 \\
\hline S3 & 27.5 & E13,E29 & 32.6 & E28 & 36.0 & E47 & 38.8 & E35 \\
\hline S4 & 31.9 & E112 & 30.3 & E20 & 31.9 & E122 & 32.4 & E41 \\
\hline Avg. \pm std. & \multicolumn{2}{|c|}{$35.9 \pm 7.4$} & \multicolumn{2}{|c|}{$41.7 \pm 11.9$} & \multicolumn{2}{|c|}{$44.6 \pm 12.1$} & \multicolumn{2}{|c|}{$46.5 \pm 12.5$} \\
\hline
\end{tabular}

Table2 shows the highest rate for the classification of the 32 syllables of Exp. I. The number of classes was four times larger than the number of initial consonants, which resulted in a reciprocal decrease in classification accuracy. Once again the SL \& EF provided the best results, except for S4, for which it yielded the rate $8.1 \%$ vs. $8.2 \%$ from EF. For this subject, the highest rates of both methods were achieved in the region of the secondary auditory cortex (A2). 
Table 2 Highest performance for the classification of the 32 syllables of Exp. I.

\begin{tabular}{|c|c|c|c|c|c|c|c|c|}
\hline \multirow[b]{2}{*}{ subject } & \multicolumn{2}{|c|}{ potential } & \multicolumn{2}{|c|}{ SL } & \multicolumn{2}{|r|}{ EF } & \multicolumn{2}{|c|}{ SL \& EF } \\
\hline & $\%$ & sensor & $\%$ & sensor & $\%$ & sensor & $\%$ & sensor \\
\hline $\mathrm{S} 1$ & 13.4 & E36 & 21.4 & E41 & 19.6 & E46 & 23.1 & E41 \\
\hline $\mathrm{S} 2$ & 9.5 & E30 & 9.9 & $\mathrm{Cz}$ & 10.9 & E35 & 11.0 & E40 \\
\hline S3 & 7.8 & E13 & 8.4 & E20 & 9.3 & E97 & 9.4 & E44,E46 \\
\hline S4 & 7.9 & E6,E13 & 7.1 & E12 & 8.2 & E116,E122 & 8.1 & $\mathrm{E} 116, \mathrm{E} 122$ \\
\hline Avg.土std. & \multicolumn{2}{|c|}{$10.0 \pm 2.5$} & \multicolumn{2}{|c|}{$12.7 \pm 6.3$} & \multicolumn{2}{|c|}{$12.8 \pm 5.0$} & \multicolumn{2}{|c|}{$14.0 \pm 6.7$} \\
\hline
\end{tabular}

Table 3 summarizes the classification result for the 4 vowels of Exp. I. The rates were significantly above the chance probability (25\%), but the highest rate $(46.9 \%, \mathrm{~S} 1)$ was significantly smaller than in the classification of the initial consonants $(63 \%, \mathrm{~S} 1)$, which had twice as many classes. Furthermore, improvements in comparison with the scalar potential were not as large in average as in the previous two cases.

Table 3 Highest performance for the classification of the 4 vowels of Exp. I.

\begin{tabular}{ccccccccc}
\hline & \multicolumn{2}{c}{ potential } & \multicolumn{2}{c}{ SL } & \multicolumn{2}{c}{ EF } & \multicolumn{2}{c}{ SL \& EF } \\
\hline subject & $\%$ & sensor & $\%$ & sensor & $\%$ & sensor & $\%$ & sensor \\
\hline S1 & 40.8 & E37 & 46.4 & E42 & 41.1 & E40 & 46.9 & E41 \\
S2 & 38.3 & E52 & 38.3 & E80 & 43.9 & E56 & 43.3 & E56 \\
S3 & 37.0 & E6 & 39.6 & E127 & 38.3 & E52 & 38.6 & E111 \\
S4 & 39.6 & E76 & 39.6 & E76 & 44.4 & E97 & 41.8 & E105 \\
\hline Avg. .std. & $39.0 \pm 1.6$ & \multicolumn{2}{c}{$41.6 \pm 3.5$} & $41.4 \pm 2.5$ & $42.8 \pm 3.4$ \\
\hline
\end{tabular}

Number of trials per participant: S1-360, S2-180, S3-316, S4-225.

Chance level: $25.0 \%$.

Table 4 shows the classification rates of Exp. II. The lowest classification rate was $52.1 \%$ for subject S2 using the surface Laplacian of the scalp potential. The highest rate $86.9 \%$ occurred for subject S1 with SL \& EF. Overall the results were remarkably good taking into account the chance probability of 11.1\%. In average, the classification rates obtained with EF and SL \& EF were much higher than those obtained with the potential and SL. The SL performed similarly to the potential in average (63.0\% vs. $61.9 \%)$ and rendered the highest standard deviation among the four methods. 
Table 4 Highest performance for the classification of the 9 images of Exp. II.

\begin{tabular}{|c|c|c|c|c|c|c|c|c|}
\hline \multirow[b]{2}{*}{ subject } & \multicolumn{2}{|c|}{ potential } & \multicolumn{2}{|c|}{ SL } & \multicolumn{2}{|c|}{ EF } & \multicolumn{2}{|c|}{ SL \& EF } \\
\hline & $\%$ & sensor & $\%$ & sensor & $\%$ & sensor & $\%$ & sensor \\
\hline S1 & 72.7 & PO8 & 81.6 & PO8 & 83.2 & $\mathrm{PO} 4$ & 86.9 & $\mathrm{PO} 8$ \\
\hline $\mathrm{S} 2^{\mathrm{a}}$ & 58.2 & O9 & 52.1 & $\mathrm{O} 2$ & 68.0 & PO4 & 71.1 & $\mathrm{O} 2$ \\
\hline S3 & 61.7 & $\mathrm{POz}$ & 60.6 & P3 & 62.2 & CP2 & 70.7 & $\mathrm{P} 3, \mathrm{P} 4$ \\
\hline $\mathrm{S} 4^{\mathrm{b}}$ & 59.5 & $\mathrm{POz}$ & 63.9 & PO8 & 68.1 & $\mathrm{O} 2$ & 75.1 & PO8 \\
\hline S5 & 66.1 & $\mathrm{PO} 3$ & 70.5 & $\mathrm{POz}$ & 76.2 & $\mathrm{P} 1$ & 81.8 & $\mathrm{POz}$ \\
\hline$S 6^{a}$ & 60.3 & $\mathrm{O} 1$ & 55.9 & $\mathrm{O} 2$ & 65.8 & CP4 & 70.0 & $\mathrm{Oz}$ \\
\hline $\mathrm{S} 7^{\mathrm{a}}$ & 54.8 & $\mathrm{Iz}$ & 56.6 & $\mathrm{P} 2$ & 67.5 & P4 & 70.6 & $\mathrm{P} 4$ \\
\hline Avg \pm std. & \multicolumn{2}{|c|}{$61.9 \pm 5.9$} & \multicolumn{2}{|c|}{$63.0 \pm 10.2$} & \multicolumn{2}{|c|}{$70.2 \pm 7.1$} & \multicolumn{2}{|c|}{$75.2 \pm 6.7$} \\
\hline
\end{tabular}

The classification rates for the trials of the mental task of Exp. III are shown in Table 5. These rates were higher than those shown in Carvalhaes and Suppes (2011) because of the averaging of trials to reduce temporal noise. Here, most of the best predictions were achieved by the tangential electric field ( $\mathrm{S} 2, \mathrm{~S} 3$, S4, S7, S9, S10, S11) rather than by SL \& EF, which yielded the highest rate for 5 participants (S3, S6, S8, S9, S11). The SL was the most accurate method for participants $\mathrm{S} 1$ and $\mathrm{S} 5$.

Table 5 Highest performance for the classification of the mental task of Exp. III.

\begin{tabular}{cclllllll}
\hline & \multicolumn{2}{c}{ potential } & \multicolumn{2}{c}{$\mathrm{SL}$} & \multicolumn{2}{c}{ EF } & \multicolumn{2}{c}{ SL \& EF } \\
\hline subject & $\%$ & sensor & $\%$ & sensor & $\%$ & sensor & $\%$ & sensor \\
\hline S1 & 95.9 & P8 & 96.7 & P8 & 95.0 & CP6 & 95.9 & P8 \\
S2 & 77.7 & FC4 & 78.5 & C4 & 83.5 & P3 & 81.8 & P1 \\
S3 & 81.0 & PO4 & 81.0 & CPz & 86.0 & P2 & 86.0 & CP4 \\
S4 & 86.7 & P7 & 82.5 & Pz & 89.2 & P1,P2 & 88.3 & P4,PO4 \\
S5 & 86.0 & P4 & 92.6 & P4 & 88.4 & CPz & 88.4 & P4 \\
S6 & 68.3 & P7,P3,PO3 & 72.5 & F7,FC3 & 73.3 & FT7 & 74.2 & Pz \\
S7 & 79.3 & PO3,O1 & 86.8 & PO3 & 87.6 & O1 & 85.1 & P3 \\
S8 & 87.6 & O1 & 81.0 & C3,C6,O2 & 90.9 & P3 & 91.7 & PO3 \\
S9 & 77.7 & C4 & 76.0 & TP9,PO3 & 80.2 & PO4 & 80.2 & PO4 \\
S10 & 88.4 & FC2,FC4 & 86.8 & P3 & 93.4 & CP3 & 92.6 & CP3 \\
S11 & 82.6 & FC2 & 86.0 & P7 & 86.8 & CP3 & 86.8 & CP5,CP3,P3 \\
\hline Avg. std. & $82.8 \pm 7.3$ & $83.7 \pm 7.1$ & $86.7 \pm 6.1$ & $86.4 \pm 6.1$
\end{tabular}


Effect sizes

We evaluated the practical significance of the results on Tables 1,5 using effect sizes and 95\% confidence intervals. The results are summarized in Table6. The tasks of classifying the 8 initial consonants and 32 syllables rendered nearly the same effect size. The classification of the 4 vowels, where the deviation from the mean classification rate was relatively small resulted in effect sizes equal to or greater than one standard deviation. An attentive reader may think that the results presented in Table 6 are at odds with those of Table 3 , where the surface Laplacian's mean classification rate was higher than that of the tangential field. We stress that such results are not inconsistent, as the effect size computation takes into account not only mean values, but also their variances. The effect sizes were all positive in Exp. II, but the confidence interval for the SL included zero, meaning that the hypothesis of no difference in performance between the potential and SL could not be rejected at $95 \%$ level of confidence. In contrast, for this experiment the superior performance of SL \& EF as compared to the potential was confirmed with 2.1 standard deviations. The effect sizes were all positive in Exp. III, but again the hypothesis of no difference between the potential and SL could not be rejected because the confidence interval included zero.

Table 6 Effect sizes and 95\% confidence intervals for improvements in classification rates.

\begin{tabular}{llll}
\hline \multicolumn{1}{c}{ Classification task } & \multicolumn{1}{c}{ SL } & \multicolumn{1}{c}{ EF } & SL \& EF \\
\hline Exp. I (initial consonants) & $0.6(0.4$ to 0.8$)$ & $0.9(0.6$ to 1.2$)$ & 1.0 (0.7 to 1.4$)$ \\
Exp. I (syllables) & $0.6(0.4$ to 0.7$)$ & $0.7(0.5$ to 0.9$)$ & 0.8 (0.6 to 1.0$)$ \\
Exp. I (vowels) & $1.0(0.6$ to 1.3$)$ & $1.2(0.6$ to 1.7$)$ & $1.4(1.0$ to 1.9$)$ \\
Exp. II & $0.1(-0.2$ to 0.3$)$ & $1.3(0.4$ to 2.1$)$ & $2.1(0.9$ to 3.4$)$ \\
Exp. III & $0.1(-0.2$ to 0.5$)$ & $0.6(0.3$ to 0.9$)$ & $0.5(0.3$ to 0.8$)$ \\
\hline
\end{tabular}

Effect of smoothing

We asked the question of whether improvements in classification rates using SL, EF, or SL \& EF were a mere consequence of the $\lambda$ regularization, instead of reflecting an intrinsic capability of these methods. If this hypothesis were true, then we should be able to improve the performance of the electric potential by classifying its regularized version. We tested this hypothesis by conducting another round of classification in which the raw potential was repeatedly 
smoothed, with $\lambda$ varying in the same log-scale grid from $\lambda=0.001$ to 100 . We compared the results with the rates obtained with the raw potential. The outcome of this analysis was that there was no significant improvement in the classification rates due to the smoothing of the potential. The largest effect size was $0.3(95 \% \mathrm{CI}, 0.2-0.5)$ and occurred in the classification of the 8 initial consonants. For all other tasks the effect size was smaller than 0.3 and the confidence interval included zero in all cases, supporting the null hypothesis of no significant effect of regularization on the classification rates.

Classification with multiple channels

We also evaluated the applicability of the electric field in multichannel classification. In principle, the use of multiple channels permits a fully exploitation of information content encoded in space and time, meanwhile accounting for correlation between channels and inter-dependent features that enlarge the number of false positives leading to misclassifications. In order to perform this evaluation using LDA, we concatenated the trials of the 5 and 10 best-performing channels disregarding their physical locations. The enlarged signals were classified using the same optimization procedure employed for single channels. Table 7 shows the resulting effect sizes. The tables showing the classification rates are presented in the Supplementary Material.

Small variations were observed in the performance of the SL, the most important one occurring in the classification of the 8 initial consonants, with a monotonic increase in effect size from 0.6 (Table6) to 0.7 and 0.8 (Table[7). The effect of the SL in the classification of the 4 vowels changed drastically, decreasing 10 folds for classification with the 10 best channels. The practical effects of EF and SL \& EF were strongly affected in the classification of the vowels, shape-color sensory images, and stop-go imagined images. They remained about the same in the two other cases, except that the effect of SL \& EF decreased from 1.0 (Table 6) to 0.5 (Table 7) in the classification of the initial consonants using 10 channels. In comparison to the single-channel classification, here the $95 \%$ confidence interval included zero in several cases, indicating loss of significance of improvements in classification rate. 
Table 7 Effect sizes for all classification tasks by concatenating the 5 and 10 best-performing channels. The columns account for the normal field, tangential field, and the total scalp electric field.

\begin{tabular}{lcccccc}
\hline & \multicolumn{3}{c}{ Five channels } & \multicolumn{3}{c}{ Ten channels } \\
\cline { 2 - 7 } \multicolumn{1}{c}{ Classification task } & SL & EF & SL \& EF & SL & EF & SL \& EF \\
\hline Exp. I (initial consonants) & 0.7 & 0.8 & 0.8 & 0.8 & 0.8 & 0.5 \\
Exp. I (syllables) & 0.7 & 0.6 & 0.8 & 0.6 & 0.8 & 0.6 \\
Exp. I (vowels) & 0.4 & 0.1 & $-0.1^{*}$ & $0.1^{*}$ & $-0.4^{*}$ & -0.9 \\
Exp. II & $-0.2^{*}$ & 0.1 & 0.4 & $0.2^{*}$ & $0.2^{*}$ & $0.3^{*}$ \\
Exp. III & $0.0^{*}$ & $-0.2^{*}$ & $-0.4^{*}$ & $0.1^{*}$ & $0.0^{*}$ & $-0.3^{*}$ \\
\hline
\end{tabular}

$* 95 \% \mathrm{Cl}$ includes zero.

\section{Discussion}

Overall the classification results were more accurate with the joint use of the surface Laplacian of the potential and the tangential electric field. The only exception was the mental task of Exp. III, for which the electric field alone was more accurate in average than any other waveform. However, this does not invalidate the view that the surface Laplacian of the potential and the tangential electric field should be used together to best assess non-overlapping information encoded in different spatial directions. Nevertheless, this exception illustrates a possible situation that may not be possible to predict, and that can be associated to particular features of our experiments.

Improvements with SL \& EF were generally less significant in multichannel classification. While the performance of the potential increased substantially with multiple channels, the other waveforms were only slightly more accurate, in some cases yielding effect sizes one standard deviation or more smaller than obtained with using single channels, and in some cases even negative effect sizes. A possible explanation for this decline in performance may be related to the way we concatenate channels for classification. Such concatenation, which has little practical effect on the nonlocal electric potential disregards the electrodes' physical locations, thus worsening the estimation of the surface Laplacian differentiation and the electric field, which are local quantities.

We recall that the locality of the electric field and the surface Laplacian was a primary reason for conducting this study. Presumably, this property should reflect in a better identification of those brain areas involved in the task performance, opening a prospect for applications on EEG brain mapping and suggesting a criterion for a prior selection of channels to perform classification. 
The three tasks studied in Exp. I were important to ascertain this hypothesis, since they involved auditory evoked activity and the signals were recorded with a high-density electrode net. In good agreement with anatomical reports (e.g., Hashimoto et al , 2000), our results with SL \& EF showed the best performing channel being close to A1 and A2 for all subjects, challenging the notion that the EEG is an unreliable detector of localized activation due to poor spatial resolution.

Recognizing vowels from syllables was the most challenging task encountered in our study. The comparatively low rates achieved in this case were possibly related to variations in the onset of vowels preceded by different consonants. As long-duration consonants take longer to be perceived as compared to short consonants, the onset of the ensuing vowel varied affecting the classification negatively. Evidence of variations in onset was reported, for instance, by Lawson and Gaillard (1981) based on the analysis of evoked potential.

Our study had several limitations that may have prevented a better assessment of the true capability of the combined use of the surface Laplacian and electric field to improve classification. The use of a spherical scalp model was one of such limitations. For instance, Babiloni et al (1996, 1998) reported significant improvements in surface Laplacian estimation by reconstructing the scalp surface with magnetic resonance (MR). Also worthy of mention is the work of He et al (2001) in which the surface Laplacian was reliably estimated using realistic electrode locations. It seems, therefore, plausible to conjecture that a similar improvement could occur here, provided that supplementary resources such as MR were available to perform spatial differentiations more accurately. Finally, we remark that numerical differentiations are inevitably affected by noise and the mechanism of $\lambda$ regularization has a limit power to prevent such effect. This limitation could be mitigated by the use of a more sophisticated statistical technique for handling noise.

\section{Conclusion}

This paper discussed the method of joint use of the surface Laplacian differentiation and tangential electric field to improve EEG classification. Its effectiveness was evaluated in the challenging problem of EEG classification using a variety of experimental conditions. In all experimental conditions (with one exception discussed above), the joint use of the surface Laplacian and tangential electric fields resulted in better classification rates for single electrode 
sites. The statistical results were quite significant in most cases, supporting a more extensive investigation of this approach in EEG analysis.

Acknowledgements We would like to thank Dr. Gary Oas and Prof. José Paulo de Mendonça for fruitful discussions on the electric field of the brain. We are also in debt to Dr. Rui Wang for her help in all matters related to Exp. I.

\section{References}

Abascal JFPJ, Arridge SR, Atkinson D, Horesh R, Fabrizi L, Lucia MD, Horesh L, Bayford RH, Holder DS (2008) Use of anisotropic modelling in electrical impedance tomography: description of method and preliminary assessment of utility in imaging brain function in the adult human head. Neuroimage 43:258-268

Babiloni F, Babiloni C, Fattorini L, Carducci F, Onorati P, Urbano A (1995) Performances of surface Laplacian estimators: A study of simulated and real scalp potential distributions. Brain Topogr 8:35-45

Babiloni F, Babiloni C, Carducci F, Fattorini L, Onorati P, Urbano A (1996) Spline Laplacian estimate of EEG potentials over a realistic magnetic resonance-constructed scalp surface model. Electroenceph Clin Neurophysiol 98:363-373

Babiloni F, Carducci F, Babiloni C, Urbano A (1998) Improved realistic Laplacian estimate of highly-sampled EEG potentials by regularization techniques. Electroenceph clin Neurophysiol 106:336-343

Babiloni C, Carducci F, Cincotti F, Rossini PM, Neuper C, Pfurtscheller G, Babiloni F (1999) Human movement-related potentials vs desynchronization of EEG alpha rhythm: a high-resolution EEG study. Neuroimage 10:658-665

Babiloni C, Babiloni F, Carducci F, Cincotti F, Percio CD, Pino GD, Maestrini S, Priori A, Tisei P, Zanetti O, Rossini PM (2000) Movement-related electroencephalographic reactivity in Alzheimer disease. Neuroimage 12:139-146

Babiloni F, Cincotti F, Bianchi L, Pirri G, del R Millï¿œn J, Mourino J, Salinari S, Marciani MG (2001) Recognition of imagined hand movements with low resolution surface Laplacian and linear classifiers. Med Eng Phys $23: 323-328$

Babiloni C, Babiloni F, Carducci F, Cincotti F, Cocozza G, Percio CD, Moretti DV, Rossini PM (2002) Human cortical electroencephalography 
(EEG) rhythms during the observation of simple aimless movements: a highresolution EEG study. Neuroimage 17:559-572

Bai O, Lin P, Vorbach S, Floeter MK, Hattori N, Hallett M (2008) A high performance sensorimotor beta rhythm-based brain-computer interface associated with human natural motor behavior. J Neural Eng 5:24-35

Becker, B J (1988) Synthesizing standardized mean change measures. Brit J Math Stat Psychol 41:257-278

Bertrand O, Perrin F, Pernier J (1985) A theoretical justification of the average reference in topographic evoked potential studies. Electroencephalogr Clin Neurophysiol 62:462-464

Besio WG, Koka K, Aakula R, Dai W (2006) Tri-Polar Concentric Ring Electrode Development for Laplacian Electroencephalography. IEEE Trans Biom Eng 53:926-933

Besio WG, Kay SM, Liu X (2009) An optimal spatial filtering electrode for brain computer interface. Conf Proc IEEE Eng Med Biol Soc 2009:3138 3141

Carvalhaes CG, Perreau-Guimaraes M, Grosenick L, Suppes P (2009) EEG classification by ICA source selection of Laplacian-filtered data. In: Proc. IEEE Int. Symp. Biomedical Imaging: From Nano to Macro ISBI '09, pp 1003-1006

Carvalhaes CG, Suppes P (2011) A spline framework for estimating the EEG surface Laplacian using the Euclidean metric. Neural Comput 23:2974-3000

Carvalhaes CG (2013) Spline interpolation on nonunisolvent sets. IMA Journal of Numerical Analysis 33:370-375

Chen GS, Lu CC, Chen CW, Ju MS, Lin CC (2005) Portable active surface Laplacian EEG sensor for real-time mu rhythms detection. Conf Proc IEEE Eng Med Biol Soc 5:5424-5426

Hashimoto R, Homae F, Nakajima K, Miyashita Y, Sakai KL (2000) Functional differentiation in the human auditory and language areas revealed by a dichotic listening task. Neuroimage 12:147-158

Haus HA, Melcher JR (1989) Electromagnetic fields and energy. Englewood Cliffs, New Jersey: Prentice Hall.

He B, Cohen, RJ (1992) Body surface Laplacian ECG mapping. IEEE Trans Biom Eng 39:1179-1191

He B, Kirby DA, Mullen TJ, Cohen RJ (1993) Body surface Laplacian mapping of cardiac excitation in intact pigs. PACE 16: 1017-1026. 
He B, Chernyak YB, Cohen RJ (1995) An equivalent body surface charge model representing three-dimensional bioelectrical activity. IEEE Trans Biom Eng 42:637-646

He B, Lian J, Li G (2001) High-resolution EEG: a new realistic geometry spline Laplacian estimation technique. Clin Neurophysiol 112:845-852

Hjorth B (1975) An on-line transformation of EEG scalp potentials into orthogonal source derivations. Electroencephalogr Clin Neurophysiol 39:526530

Jolliffe I (2005) Principal component analysis. John Wiley \& Sons, Ltd.

Kayser J, Tenke CE (2006a) Principal components analysis of Laplacian waveforms as a generic method for identifying ERP generator patterns: I. Evaluation with auditory oddball tasks. Clin Neurophysiol 117:348-368

Kayser J, Tenke CE (2006b) Principal components analysis of Laplacian waveforms as a generic method for identifying ERP generator patterns: II. Adequacy of low-density estimates. Clin Neurophysiol 117:369-380

Koka K, Besio WG (2007) Improvement of spatial selectivity and decrease of mutual information of tri-polar concentric ring electrodes. J Neurosci Meth $165: 216-222$

Lawson EA, Gaillard AW (1981) Evoked potentials to consonant-vowel syllables. Acta Psychol (Amst) 49:17-25

Mikkulainen R (2005) Computational maps in the visual cortex, Springer, New York

Murray MM, Brunet D, Michel CM (2008) Topographic ERP analyses: a stepby-step tutorial review. Brain Topogr 20:249-264

Nakagawa S, Cuthill IC (2007) Effect size, confidence interval and statistical significance: a practical guide for biologists. Biol Rev Camb Philos Soc 82:591-605

Nunez PL, Pilgreen KL (1991) The spline-Laplacian in clinical neurophysiology: a method to improve EEG spatial resolution. J Clin Neurophysiol 8:397-413

Nunez PL, Pilgreen KL, Westdorp AF, Law SK, Nelson AV (1991) A visual study of surface potentials and Laplacians due to distributed neocortical sources: computer simulations and evoked potentials. Brain Topogr 4:151168

Nunez PL, Silberstein RB, Cadusch PJ, Wijesinghe RS, Westdorp AF, Srinivasan R (1994) A theoretical and experimental study of high resolution EEG based on surface Laplacians and cortical imaging. Electroencephalogr Clin 
Neurophysiol 90:40-57

Nunez PL, Westdorp AF (1994) The surface Laplacian, high resolution EEG and controversies. Brain Topogr 6:221-226

Nunez PL, Srinivasan R (2006) Electric Fields of the Brain: The Neurophysics of EEG, 2nd edn. Oxford University Press, New York

Oostenveld R, Praamstra P (2001) The five percent electrode system for highresolution EEG and ERP measurements. Clinical Neurophysiology 112:713719

Parra LC, Christoforou C, Gerson AD, Dyrholm M, Luo A, Wagner M, Philiastides MG, Sajda P (2008) Spatiotemporal linear decoding of brain state. Signal Proc Mag, IEEE 25:107-115

Perrin F, Pernier J, Bertrand O, Echallier JF (1989) Spherical splines for scalp potential and current density mapping. Electroencephalogr Clin Neurophysiol 72:184-187

Petrov Y (2012) Anisotropic spherical head model and its application to imaging electric activity of the brain. Phys Rev E 86:011,917

Pickles JO (2008) An introduction to the physiology of hearing, 4th ed. Bingley, U.K., Emerald

Plonsey R (1969) Bioelectric Phenomena. McGraw-Hill, New York

Srinivasan R, Nunez PL, Tucker DM, Silberstein RB, Cadusch PJ (1996) Spatial sampling and filtering of EEG with spline Laplacians to estimate cortical potentials. Brain Topogr 8:355-366

Suppes P, Perreau-Guimaraes M, Wong DK (2009) Partial orders of similarity differences invariant between EEG-recorded brain and perceptual representations of language. Neural Comput 21:3228-3269

Wahba G (1990) Spline models for observational data. Philadelphia: SIAM

Wang R (2011) Recognizing phonemes and their distinctive features in the brain. PhD thesis, Stanford University

Wang R, Perreau-Guimaraes M, Carvalhaes C, Suppes P (2012) Using phase to recognize English phonemes and their distinctive features in the brain. Proc Nac Acad Sci 109:20685-20690.

Yao D (2002) High-resolution EEG mapping: a radial-basis function based approach to the scalp Laplacian estimate. Clin Neurophysiol 113: 956-967 
Appendix

This appendix provides an intuitive explanation for the relation between the surface Laplacian of the scalp potential and the normal component of the scalp electric field when recording the EEG. For clarity of exposition, we focus on the current density $\mathbf{J}$, which is a vector quantity that is locally related to the electric field $\mathbf{E}$ in the extracellular space by $\mathbf{J}=\sigma \mathbf{E}$, where $\sigma$ is the electrical conductivity of the medium. The current density is quasistatic continuous (e.g., Plonsey, 1969; Haus and Melcher, 1989), thus obeying $\nabla \cdot \mathbf{J}=0$. Using spherical coordinates, this equation can be expressed in the form

$$
\frac{1}{r^{2}} \frac{\partial}{\partial r}\left(r^{2} J_{r}\right)+\frac{1}{r \sin \theta} \frac{\partial}{\partial \theta}\left(\sin \theta J_{\theta}\right)+\frac{1}{r \sin \theta} \frac{\partial J_{\varphi}}{\partial \varphi}=0 .
$$

We can conveniently rewrite this equation as

$$
\nabla_{s} \cdot \mathbf{J}_{s}=-\frac{2}{r} J_{r}-\frac{\partial J_{r}}{\partial r}
$$

where $\nabla_{s} \cdot \mathbf{J}_{s}$ is the surface divergence of the tangential current density $\mathbf{J}_{s}=J_{\theta} \hat{\boldsymbol{\theta}}+J_{\varphi} \hat{\boldsymbol{\varphi}}$.

The term $(2 / r) J_{r}$ in the right-hand side of equation (11) represents the contribution of the spherical geometry to lateral spread of current. This term vanishes as $r$ goes to infinity, where the sphere looks locally like a plane and the geometry does not affect current flow in the normal direction. This limit corresponds to the model studied by He and Cohen (1992) and He et al (1995). The term $\partial J_{r} / \partial r$ represents the rate of vanishing of $J_{r}$ in the normal direction, and is particularly significant at the scalp-air interface, where the negligibility of the air conductivity causes the abrupt vanishing of $J_{r}$ along the outer scalp surface. Hence, an intuitive interpretation of equation (11) is that it describes how the geometry and changes in flux in the normal direction affect the behavior of $\mathbf{J}_{s}$, so as to ensure the continuity of the total current density.

Both terms on the right-hand side of equation (11) depend on boundary conditions. Assuming that the scalp is surrounded by air implies the vanishing of $J_{r}$ along the outer scalp surface due to the negligibility of the air conductivity, which is about 14 orders of magnitude smaller than the scalp conductivity and prevents current to exit the head through the scalp-air interface. The recording of EEG signal changes this condition locally, causing, inevitably, an outflow of current beneath the measurement electrodes. Presumably, without the constraint of the abrupt vanishing of $J_{r}$ the magnitude of $\partial J_{r} / \partial r$ becomes smaller at these locations, so that, at least to the lowest order of approximation, we can use (11) to write

$$
\nabla_{s} \cdot \mathbf{J}_{s}(\mathbf{r}) \approx-\frac{2}{r_{\text {scalp }}} J_{r}(\mathbf{r}),
$$

it being understood that the position $\mathbf{r}$ coincides with a scalp electrode location.

Let $\sigma_{s}^{\text {scalp }}$ and $\sigma_{r}^{\text {scalp }}$ represent the tangential conductivity and the radial conductivity along the scalp. Substituting $J_{r}=\sigma_{r}^{\text {scalp }} E_{r}^{\text {scalp }}$ and $\mathbf{J}_{s}=\sigma_{s}^{\text {scalp }} \mathbf{E}_{s}^{\text {scalp }}=-\sigma_{s} \nabla_{s} \Phi_{s}^{\text {scalp }}$, where $\nabla_{s} \Phi_{s}^{\text {scalp }}$ is the surface gradient of the surface potential $\Phi_{s}^{\text {scalp }}$, and assuming that the conductivities $\sigma_{r}^{\text {scalp }}$ and $\sigma_{s}^{\text {scalp }}$ are approximately constants, we obtain from (12)

$$
E_{r}^{\text {scalp }}(\mathbf{r}) \approx \frac{r_{\text {scalp }}}{2} \frac{\sigma_{s}^{\text {scalp }}}{\sigma_{r}^{\text {scalp }}} \nabla_{s}^{2} \Phi_{s}^{\text {scalp }}(\mathbf{r})
$$


Since $E_{r}^{\text {scalp }}$ is locally related to $J_{r}^{\text {scalp }}$, this expression agrees with the usual view that the surface Laplacian differentiation provides a good method to associate local EEG events generated by cortical radial dipoles to the underlying physical structure. But the approximation (13) was obtained without any assumption about brain sources.

The scalp tangential conductivity $\sigma_{s}^{\text {scalp }}$ and the radial conductivity $\sigma_{r}^{\text {scalp }}$ were introduced to account for a prominent directional dependency in the scalp structure, as pointed out by experimental studies (e.g., Abascal et al, 2008; Petrov, 2012). Accounting for this anisotropy requires a tensor representation for $\sigma^{\text {scalp }}$, which in our model was written

$$
\boldsymbol{\sigma}^{\text {scalp }}=\sigma_{r}^{\text {scalp }} \hat{\mathbf{r}} \hat{\mathbf{r}}+\sigma_{s}^{\text {scalp }} \hat{\boldsymbol{\theta}} \hat{\boldsymbol{\theta}}+\sigma_{s}^{\text {scalp }} \hat{\boldsymbol{\varphi}} \hat{\boldsymbol{\varphi}}
$$

Typically, the ratio $\sigma_{s}^{\text {scalp }} / \sigma_{r}^{\text {scalp }}$ is about 1.5 , so that the multiplying factor $r_{\text {scalp }} \sigma_{s}^{\text {scalp }} / 2 \sigma_{r}^{\text {scalp }}$ in (13) is approximately $7.0 \mathrm{~cm}$ for typical values of the head radius. 